\title{
Speech Therapy, New Developments and Results in LingWare
}

\author{
Wolfgang Grießl, F.J. Stachowiak \\ c/o Phoenix Software, Küdinghovener Straße 98, D-53227 Bonn, \\ (0049)(0)228/97584-0 (Tel.), (0049)(0)228/07584-18 (Fax)
}

\begin{abstract}
LingWare/STACH is a permanently extended multimedia program for computer assisted language and speech therapy, which includes besides the therapy programme an author system for creative and extensive use. A short example of one of the more than 2000 exercises is given.
\end{abstract}

Since two years LingWare is extended: new soundcards, foreign versions, features to support home based therapy. The principles are outlined.

The German Federal Ministry of Research and Technology provided financial support to carry out a randomized multicenter study on the efficacy of LingWare/STACH as a form of supplementary therapy in addition o the language therapy provided by a speech therapist. A detailed description of the study and the results is given.

\section{Speech Therapy with LingWare/STACH}

LingWare/STACH was developed since 1985 and used is in a randomized multicentric study. The therapy system, in the meantime in clinical use at more than 400 facilities, is able to synchronize graphics (e.g. scanned pictures of daily situations), written and spoken text (by means of a soundcard)), thereby allowing the presentation of interactive exercises on the monitor.

For instance patients learn the correct use of prepositions from a multiple choice set of prepositions which fits in the sentence accompanying a picture. After the exercise desired is selected, the first picture in a series of pictures appears (e.g. a man is standing on a ladder). The sentence The man ist standing ... the ladder ent ers the screen from the right (Figure 1).

The multiple set of prepositions is given at the top of the screen. The instructions in dialogue line ask the patient to move the cursor to the correct preposition. The patient controls the movement of the cursor by a joystick, mouse, trackball or the cursor keys. The dialogue line alerts the patient to an incorrect answer. This is fortified by speech output: Sorry incorrect The dialogue line ask to try again.

When the correct preposition has been chosen, the complete sentence is shown along with the corresponding speech output. In the case of erroneous performance the 
computer can provide cues in a form of easier tasks, e.g. the presentation of a situation in which the prepositons are visually represented or a naming task, etc..

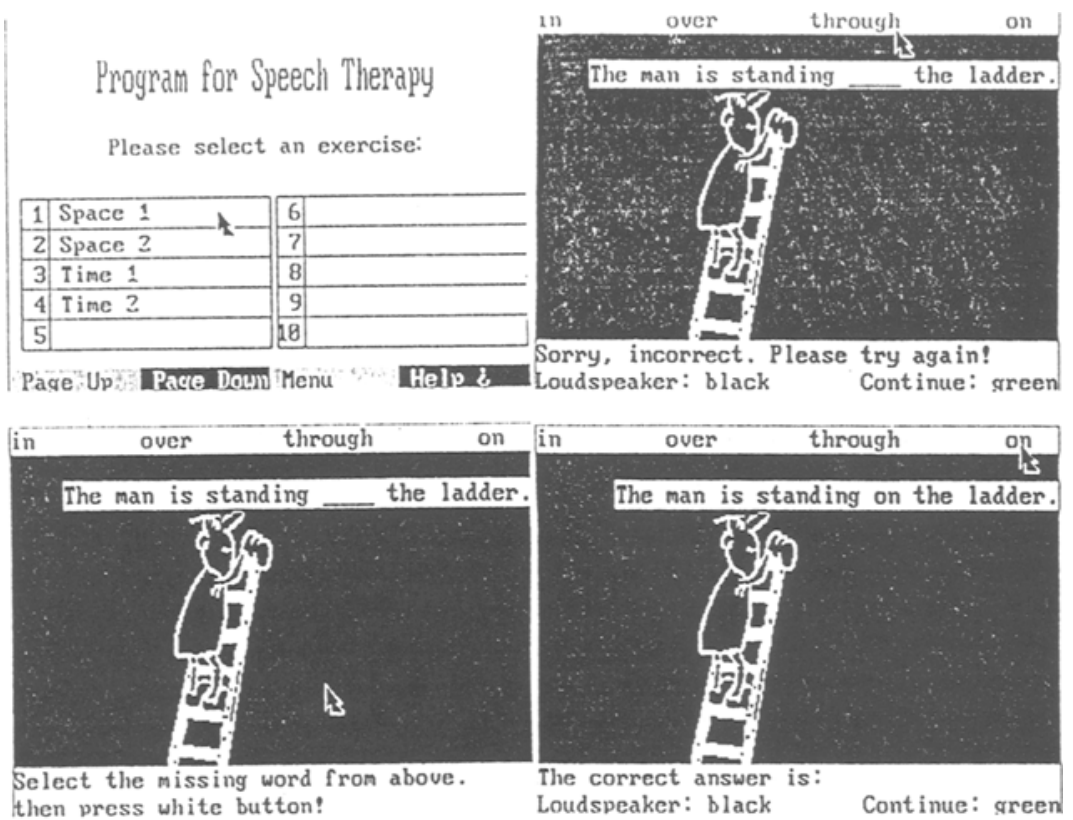

Figure 1: Exercise example

There is a total of more than 2000 exercises, based on 20 different open exercise schemes. The exercises comprise language modalities such as naming, dictation, sentence structure, comprehension, sound discrimination.

\section{Home Based Speech Therapy}

A lot of patients would be able to work with a therapy computer at home - without the permanent support by therapist. Some requests must be fulfilled by the system to offer home based computer therapy in addition to the conventional therapy:

The responsible therapist must be able to construct subsets of the total amount of exercises according to the special need of the patients. We introduced in our system 
the feature of therapy plan. Once a set of therapy plans is constructed, each therapist is responsible to assign a well suited therapy plan to each patient.

Different user interfaces must be available for therapists and patients - according to the special needs of each user group. We introduced in our system the subsystem user managment. A therapist as a user has now available all the powerful facilities of the system (author system, user management, statistics, therapy management, etc.), a patient as user only sees his assigned exercises in his therapy plan.

It must be possible to copy the patients exercises onto a random access medium. Using his volume, each patient can do his homework outside the clinical institution, for example at home. Now our system is able to copy therapy plans onto floppy discs. The statistical data of each patient are stored also on that floppy disc - the statistical analysis can be done by the therapist in his clinical institution. To decrease the system cost for patients version, the newer versions of LingWare/STACH now support the worldwide available soundblaster card.

To increase the patients motivation again it should be more easier to include private therapy material in the computer-based speech therapy: pictures of holidays, hobbies, family, friends, etc.. The newest version of our system now supports the use of video cameras together with videoblaster adapters. The standard material which comes with the system is now stored on a CD-ROM, the new private material is stored on harddiscs or floppies.

\section{Evaluation of Computer Assisted Speech Therapy}

The German Federal Ministry for Research and Technology (BMFT) supported a study examining the effects of supplementary computer assisted speech therapy performed with the LingWare/STACH system as a supplementary form of training.

The purpose of the study was not to compare computer assisted training to therapy performed by a professional speech therapist. The computer was considered to be a tool of speech therapy. The question examined was: Is the computer-based therapy effective as a form of supplementary speech therapy? The patients were devided into two groups: one group received one hour of conventional therapy per day and the other received 30 minutes conventional therapy and 30 minutes of supplementary computer assisted speech therapy per day.

As the literature (Baso, 1987) had stated that effects of speech therapy are almost impossible to show, if the patients have not been treated daily for a period of at least 6 weeks. The study embodied a six-week training period, i.e. thirty hours of language training between pre- and post-testing with the Aachen Aphasia Test Battery (AAT). The patients were assigned randomly to one of the two experimental groups, using a strict randomization procedure. The effect of spontaneous remission was controlled by including only patients with at least four months post-onset. 


\begin{tabular}{|l|l|}
\hline 2 therapy groups & $\begin{array}{l}\text { speech therapy versus speech therapy plus } \\
\text { supplementary computer training }\end{array}$ \\
\hline Length of treatment & 6 weeks \\
\hline Length of study & 24 months \\
\hline Multi-centric & 12 centers involved \\
\hline Grouping & randomized \\
\hline Stratification & according to center and degree of severity \\
\hline Testing & pre and post therapy \\
\hline Scale & $\begin{array}{l}\text { AAT, Token Test, evaluation of spontaneous } \\
\text { language }\end{array}$ \\
\hline
\end{tabular}

Figure 2: Study concept

Other exclusion criteria were double sided brain damage and progressive brain damage such as senile dementia, etc.. Biometric procedures put together groups which were comparable in age, sex and other factors. The study was limited to patients who reached the second part of the Token Test, meaning, that they had at least a minimum of language capacity.

It is clear that this study, which for statistical reasons has to include at least 150 patients, would answer the general question on the supplementary computer training as far as this was possible with standardized testing procedures such as the AAT. It is generally recognized, that the AAT is a relatively crude instrument in that it measures performance in language modalities such as repetition, naming, language comprehension, etc., but does not measure other factores relevant to therapy such as verbal short term memory, the speed of language processing and attention as well as linguistic factors such as syntactic and semantic areas of language competence and performance. Furthermore, it does not measure communication abilities except in Part 1 (Spontaneous Language). Nevertheless, we hoped that the AAT would show at leat tendencies of the effects of supplementary computer training.

\section{The Study Population}

156 aphasic patients were recruited for this study. Everyone gave her/his written consent to participate in the six-weeks training and for the data to be analyzed. Almost all the patients were in-patients at rehabilitation centres during the participation in the study.

77 patients received conventional speech therapy, 79 conventional speech therapy plus supplementary computer training. The patients did not work independently at the computer; a therapist or trained volunteer was present at each session to assist the patients in selecting exercises and operating the programme as far as necessary. The data was recorded automatically by the computer. 


\begin{tabular}{|l|l|}
\hline Inclusion Criteria & Exclusion Criteria \\
\hline Aphasic, according to AAT & Age $>75$ years \\
\hline Duration of illness $>6$ months & Bilateral lesion \\
\hline & retrogade anterogade amnesias \\
\hline & progredient illness \\
\hline & $\begin{array}{l}\text { not reaching the second part of the } \\
\text { Tokentest }\end{array}$ \\
\hline & Score of $<6$ on the Tokentest \\
\hline & Inability to work with a computer \\
\hline
\end{tabular}

Figure 3: Selection criteria

Ten patients dropped out due to illness or technical difficulties at one of the participating centres, respectively. One patient dropped out at his own wish, as he did not enjoy this form of training.

\section{Results}

All of the patients were routinely examined with the AAT. Therapist and examiner were usually not identical. The Biometrisches Zentrum Aachen (BZA) controlled the data collection and carried out the statistical analysis. Figure 4 gives an overview of the raw scores reached in the subtests of the AAT.

The scores are given for both groups and for the subtests: Tokentest, Repetition (Nachsprechen), Written language (Schriftsprache), Naming (Benennen), Oral and written comprehension (Sprachverständnis). Every dot stands for a patient and shows the number of points more or fewer achieved in the post-test as compared to pre-test scores. For instance the highest score (25) in the subtest repetition for the computer group shows that this patient improved by 25 points over the pre-test score. The lowest score $(-15)$ shows that this patients score worsened by 15 points following the 6-week training. Altogether this figure shows that most of the patients scored above the zero line, demonstrating that most patients showed improvements in the AAT following therapy.

The clearest differences were in written language and naming, which were also the focal points of the computer training programme.

The AAT (see Huber et al, 1983) gives criteria for establishing an improvement as being significant for an individual patient. According to these criteria the results were evaluated for each individual patient: Figures 4-7 show how many patients from each syndrome group showed significant improvements in the five subtests for the computer group and the conventional groups, respectively. Thus in comparison, 10 of 20 Broca aphasics in the computer group and 5 of 29 Broca aphasics in the conventional group showed significant improvements in the Tokentest. 


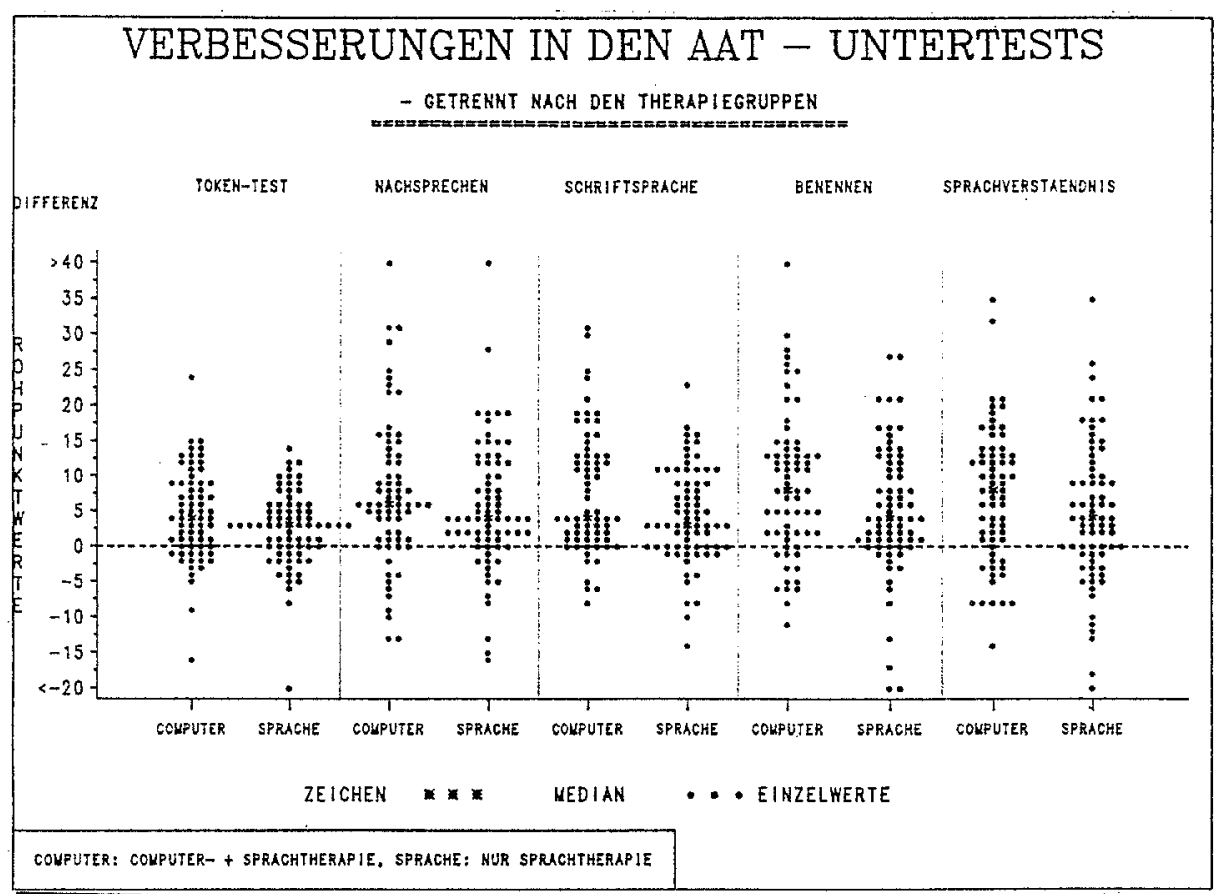

Figure 4: Improvements in the AAT - Subtests

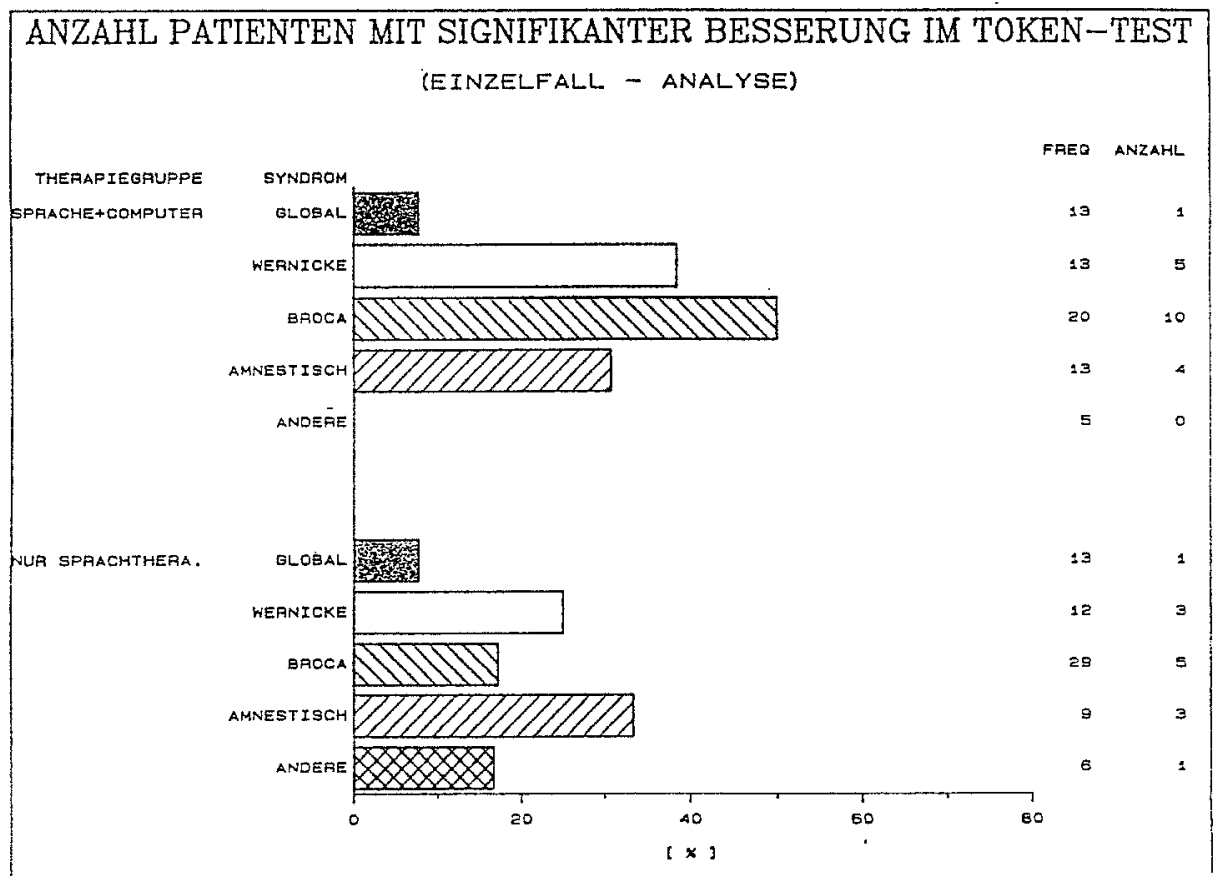

Figure 5: Improvements in Tokentest 


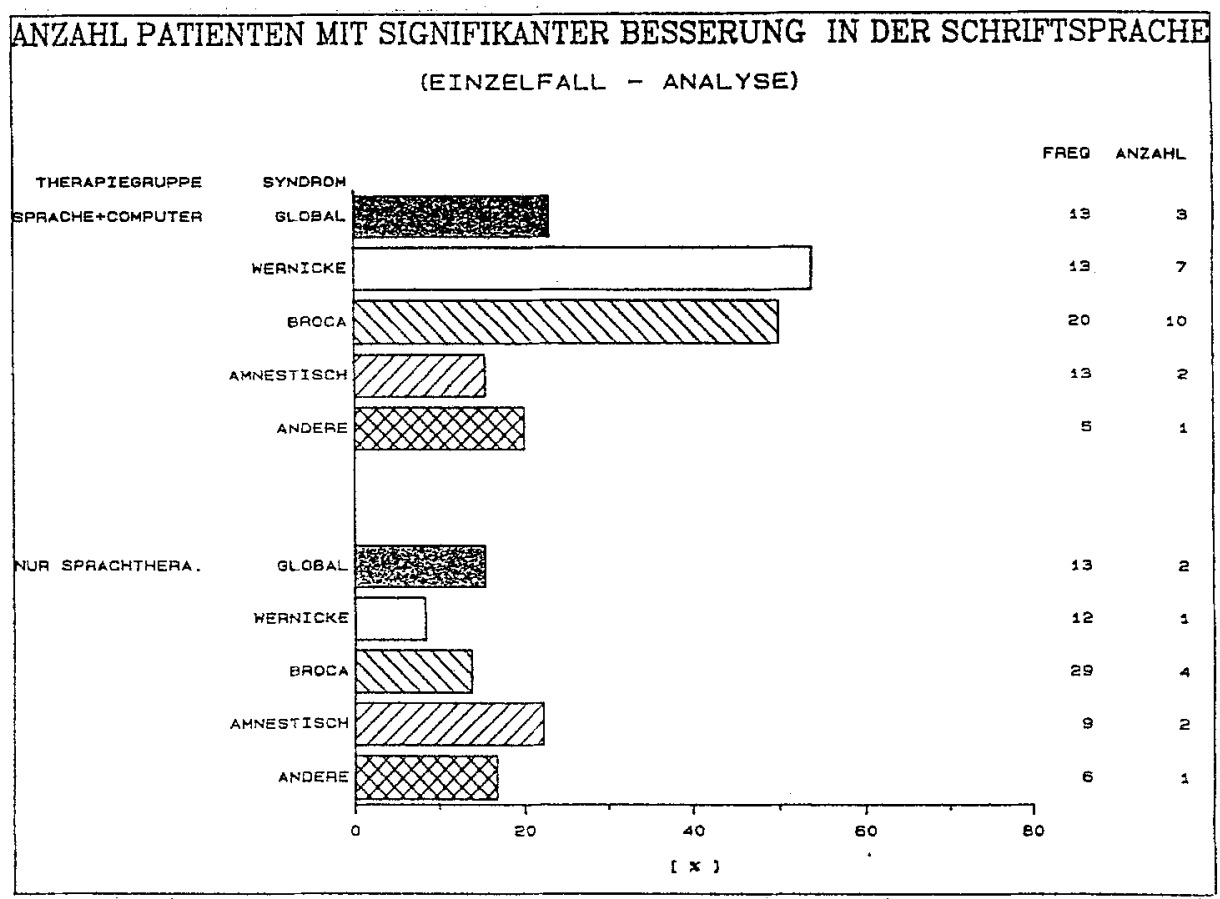

Figure 6: Improvements in written language

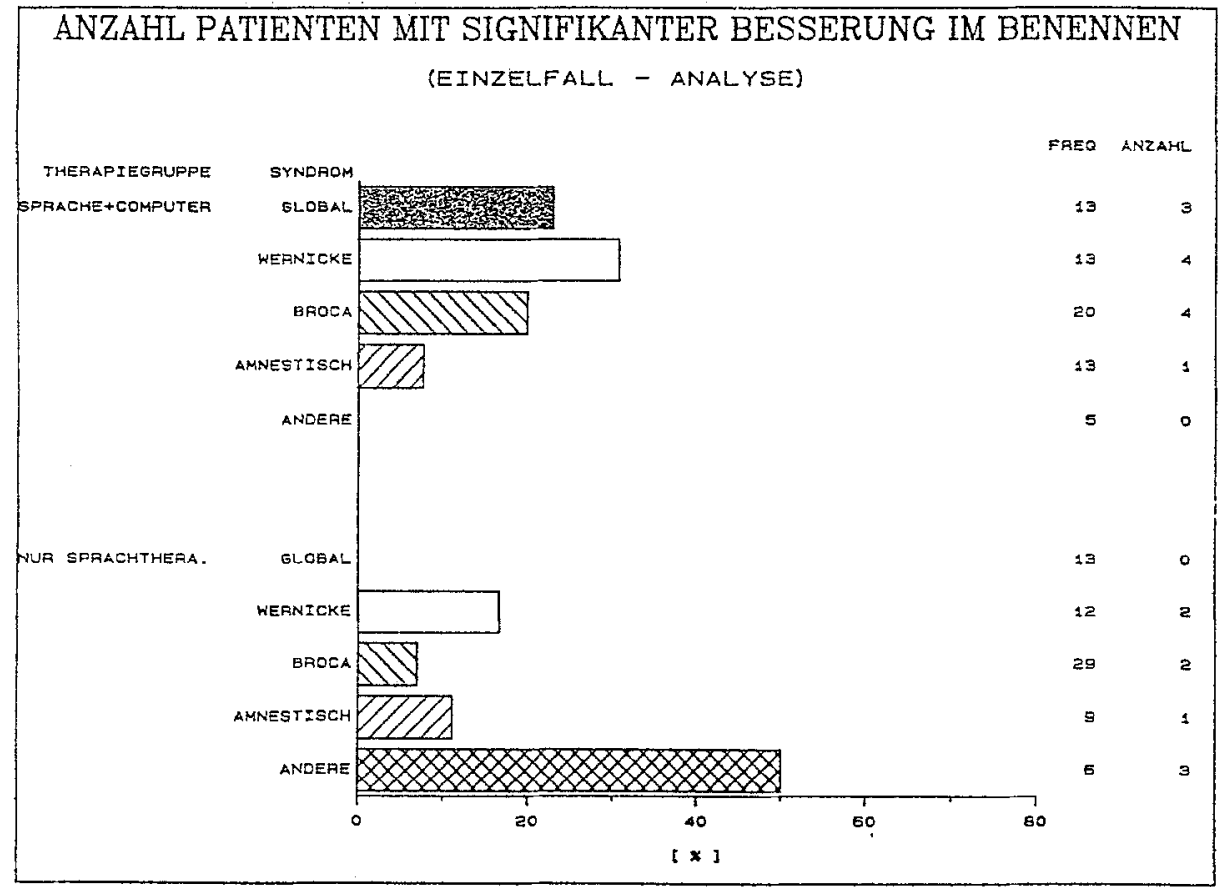

Figure 7: Improvements in naming 
Figure 6 shows the high proportion of Broca's and Wernicke's patients with significant improvements in the subtest for written naming in the computer group. Here single case analysis clarifies the results obtained by simply comparing the raw scores in Figure 4.

The evaluation of spontaneous language in the AAT also showed better effects for the computer group. A difference of $45.3 \%$ as compared to $30.4 \%$ (percentage of patients who improved by at least one point on the AAT for communicative behaviour) was observed.

\section{Discussion}

It must be emphasized that progress in therapy was not measured by the material trained, but with a standardized test measuring performance in certain language modalities. Practically speaking, this corresponds to a generaliziation effect to untrained material.

If the results are summarized, we first see positive effects of speech therapy in general and secondly effects of supplementary computer training focusing on naming and written language. This is especially true for Broca and Wernicke aphasics and more for women than for men.

A post hoc study with 17 patients also showed, that the same amount of training received over a period of 12 weeks did not achieve the same effects. These speaks in favour of a cost-reduction effect.

Taking into consideration the wide acceptance of the programme by patients and therapists and the supplementary effects combined with potential effects from a sixweek stationary treatment, the results are promising.

\section{References}

Basso, A. (1987), "Approaches to Neuropsychological Rehabilitation: Language Disorders" in: M.J. Meier et.al. (eds.): Neuropsychological Rehabilitation. Edinburgh, Churchill Livingstone, 294 - 314.)

Grieß1, W, (1994), "LingWare-Therapiesystem", User Manual, Phoenix Software GrmbH, Bonn

Grieß1, W., Stachowiak, F.J., "Computer Assisted Speech Therapy" in: W.L. Zagler (ed.): 3rd ICCHP, Wien, 1992

Huber, W., Poeck, K., Weniger, D. \& Willmes, K. (1983), Aachener Aphasie Test (AAT). Göttingen, Hogrefe 